\title{
HIGHLY SPACE-EFFICIENT ELECTROSTATIC ZIGZAG TRANSMISSIVE MICRO-OPTIC SWITCHES FOR AN INTEGRATED MEMS OPTICAL DISPLAY SYSTEM
}

\author{
Kerwin Wang1, Karl F. Böhringer ${ }^{1}$, Mike Sinclair ${ }^{2}$, and Gary Starkweather ${ }^{2}$ \\ 1University of Washington, Seattle, WA 98195, USA, kerwin@ee.washington.edu \\ ${ }^{2}$ Microsoft Research, Redmond, WA 98052, USA
}

\begin{abstract}
This paper presents likely the world's smallest electrostatic visible light highly space-efficient transmissive microoptical switches (TMOS) for an integrated MEMS optical display system. Each TMOS represents one pixel with $150 \mu \mathrm{m} \times 150 \mu \mathrm{m}$ spacing in a display module. It is demonstrated that a zigzag electrostatic actuator of $47 \mu \mathrm{m} \times 160 \mu \mathrm{m}$ size can achieve a maximum static lateral displacement of $10 \mu \mathrm{m}$ at $38 \mathrm{~V}$. Depending on the zigzag actuator design, these devices have a mechanical resonance frequency of up to $18.6 \mathrm{kHz}$.
\end{abstract}

Keywords: Electrostatic actuator, Transmissive, Opticswitch, Space-efficient, Display, MOEMS

\section{INTRODUCTION}

Several optical MEMS based display technologies have been proposed such as DLP [1,2], GLV [3], IMod [4], Gyricon [5], among others. Most of these technologies are based on reflective optics. However, their reflective nature limits their working environments, posing constraints on light intesity and contrast. A TMOS can intercept a focused

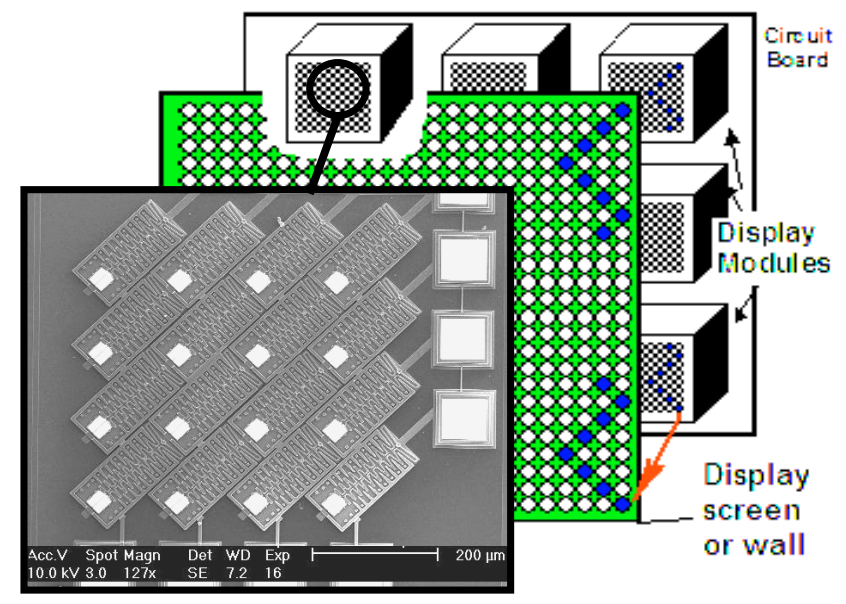

Figure 1. A high contrast, low power consumption, highly space-efficient transmissive optical switch is at the heart of the integrated MEMS optical display system. light beam in free space and thus achieves near-ideal contrast ratios, making it one of the best candidates for bright, efficient, and high contrast displays (Figure 1). It also can be used in various other applications such as, e.g., photodynamic therapy instrumentation, data storage, or optical networks.

Since the actuators for a transmissive display cannot share their working space with the light path, it is a challenge to design a highly space-efficient TMOS. There are many elegant transmissive optical switches, e.g. [6,7]. However, existing prototype transmissive optical switches generally are too space-consuming to be integrated into display arrays with sufficiently high resolution. None of them satisfies the optical design criteria (able to yield a $20 \mu \mathrm{m}^{2}$ opening within $150 \mu \mathrm{m}^{2}$ device area.) Critical actuator design goals are thus small size, large shutter opening, and high speed. This paper proposes the zigzag electrostatic actuator pair with shutters for the TMOS.

\section{OPTICAL DESIGN}

The TMOS consists of an electrostatic "zigzag" actuator pair, overlapping shutters and a miniaturized optical tunnel. The dimensions of these three components are mainly depending on the optical configuration. A microlens array can focus light into the aperture to minimize the optical loss. A second microlens array placed behind the TMOS array will image the light onto a display screen area or a projector lens. Assuming the light presented to the first microlens array has a nearly planar wave front, then each microlens focuses its portion of the light into the aperture adjacent to the shutter (Figure 2). The shutter can modulate the light by controlling

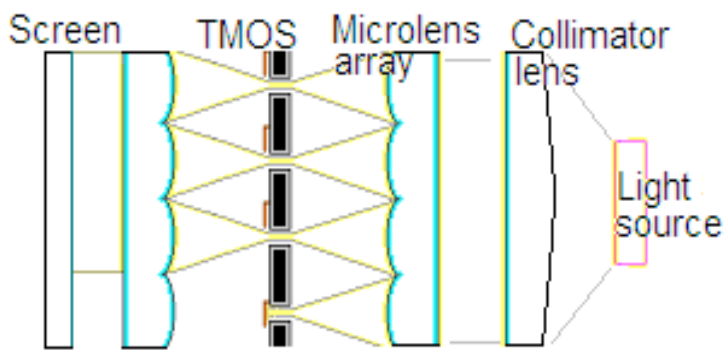

Figure 2. The microlens array can focus light into the aperture to minimize the optical loss. 
its opening. A small spot size only needs a small shutter and movement to fully turn the light on and off. It also implies low driving voltage, low power consumption, and higher frequency operations. The minimal spot size and the profile of the optical tunnel are mainly constrained by the depth of focus, diffraction spot size and the numerical aperture of the selected microlens system. These criteria should be taken into consideration to minimize the optic loss in TMOS design. Under the definition of the diffraction depth of focus $D O F=4 \lambda(f / D)^{2}$, the substrate thickness $t$ should be smaller than $1 / 2 D O F=2 \lambda(f / D)^{2}$. For $10<f / D<16$ lens systems, within visible wavelengths, a substrate thickness of $150 \mu \mathrm{m}$ will be sufficiently thin. If the light is from a diffractionlimited lens, then the diffraction spot size $D S$ is defined as: $D S=2.44 \lambda f / D, D$ is aperture. $f / D$ is the focal number or focal ratio of the lens. If $\lambda=0.76 \mu \mathrm{m}$ and $f / D=10, D S=$ $18.5 \mu \mathrm{m}^{2}$, then the minmal shutter openning should be greater than $18.5 \mu \mathrm{m}^{2}$.

Assuming the pixel size equal to TMOS, a $150 \mu \mathrm{m} \times 150 \mu \mathrm{m}$ spacing TMOS array will yield a 15-inch single color HDTV monitor. Based on these optical design goals and constraints, the shutter and the actuators should be arranged into the TMOS unit and yield at least an $18.5 \mu \mathrm{m}^{2}$ opening.

\section{ACTUATOR DESIGN}

Design of a highly space-efficient electrostatic actuator for TMOS is challenging since most electrostatic actuators, such as comb drives, take up a large area for combs and long suspending springs to provide high force/stiffness ratios for larger displacement [6]. Using a smaller gap can yield larger forces but also decreases the space for displacement. Recently, several approaches have been made including curved structures [7,8], engagement comb drives [9], and optimal shape design of a comb [10]. The curved electrode structures have gradually increasing gaps, which

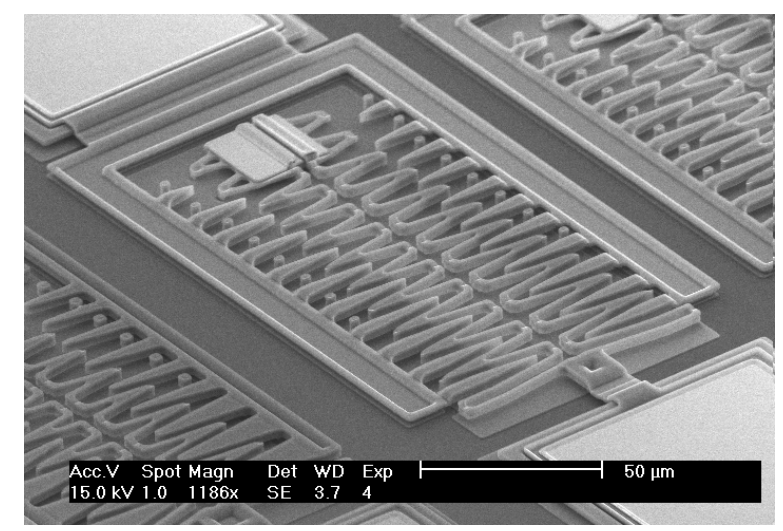

Figure 3. SEM of one TMOS confined to $106 \mu \mathrm{m} \times 188 \mu \mathrm{m}$ area, which fits a $150 \mu \mathrm{m} \times 150 \mu \mathrm{m}$ lens array. It consists of dual zigzag actuators; each takes $47 \mu \mathrm{m} \times 160 \mu \mathrm{m}$ of space. The bumpers, assisted by PECVD carbon fluoride polymer coating, prevent short circuits. The driving voltage is applied to the teeth and the zigzags to move the shutter. The detailed features of the shutter are shown in Figure 4. significantly increase the displacement, but they still require more area or higher voltages. This paper proposes a new design: the zigzag actuator (Figure 3). Each zigzag consists of 10-11 teeth. The teeth works both like an extended flexible spring and shape modified comb. It can make very efficient use of the available space by simultaneously increasing the driving force and decreasing the spring stiffness. There are two zigzags that have been placed into the TMOS; each one can take care of half of the motion according to the opening requirement. The sizes of the teeth are gradually decreased towards the free ends to allow larger shutter motion with low driving voltages. A novel design concept based on the fringing effect also has been investigated.

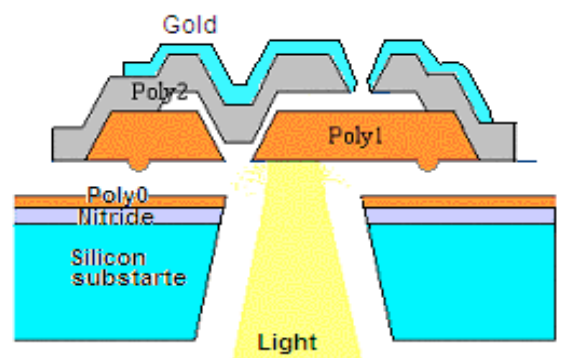

Figure 4. The cross section of a shutter; made from Poly1, Poly2 and Gold. The masses of its two components are equal for stable dynamic performance. The shutter with $2 \mu \mathrm{m}$ mutual overlap is covered by $0.5 \mu \mathrm{m}$ gold as opaque material. The dimples work together with PECVD carbon fluoride coating to reduce sticking. The channel is dry etched by DRIE and RIE for light transmission. Figure 7 shows that the shutter can successfully switch the light on and off.

\section{FRINGE EFFECT}

Fringe effects usually are not desired in sensor design because they cause non-linearities. But fringing may be useful in actuator designs to increase the electrostatic force/stiffness ratio. In high electrode-surface-area/gap ratio electrodes or comb drives, the capacitance can be modeled by a parallel-plate capacitor. When we increase the height (electrode, beam, and comb) the force/stiffness ratio will remain constant, but when the thickness is reduced towards the geometry limits, then this ratio is no longer constant. Capacitance will be higher than calculated from the simplified parallel-plate model. This is because of the fringe effect.

Maxwell ${ }^{\circledR}$ simulation results show that different thickness combinations of zigzag structures and electrodes (Figure 5) allow us to exploit fringing effects and to reduce the driving voltage. Under the same applied voltage and gap, the single height zigzag will have more deflection than the double height structure because of the higher force/stiffness ratio.

When the height of the beam decreases from double height (Poly1+Poly2) to single height (Poly1), the stiffness will 
decrease $43 \%$ but the electrostatic force will only decrease $28 \%$. Thus, under the same applied voltage and gap, the single height beam will have more deflection than the double height structure.

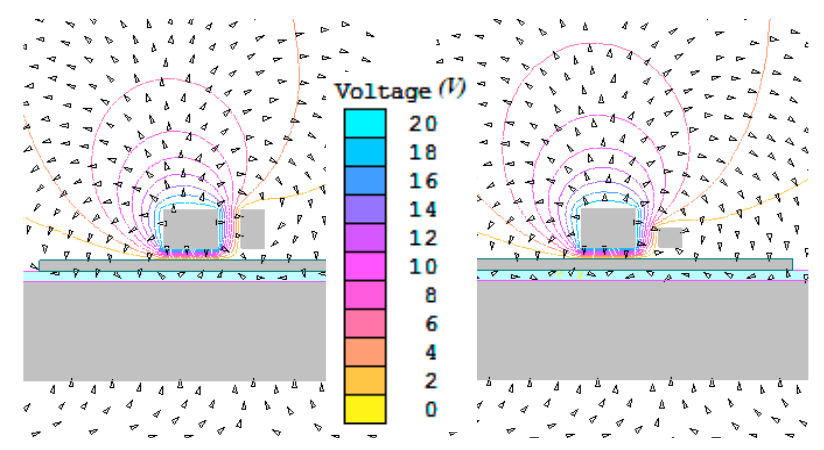

Figure 5. 2-D Maxwell ${ }^{\circledR}$ simulation results: The image shows potential contour and electrical field of double (left) and single (right) beams with $20 \mathrm{~V}$ difference.

\section{DEVICE FABRICATION}

The prototypes were fabricated in Cronos MUMPs ${ }^{\circledR}$, with post-processing developed at the University of Washington. The purpose of the post processes is to open optical tunnels and adjust apertures to fit various microlens systems. Processing included backside mechanical and chemical polishing (chip thinning), double-side-aligned DRIE based optical tunnel etching, RIE (aperture adjusting etch), HF sacrificial oxide removal with supercritical point drying, and PECVD $\left(\mathrm{C}_{3} \mathrm{~F}_{6}\right)$ hydrophobic fluorocarbon polymer coating.

The backside of an unreleased MUMPs die was first polished mechanically by a 600 grade $\mathrm{SiC}$ to remove the material (deposited polysilicon and oxide in MUMPs) from the unpolished backside. The chip-thinning etching was completed by wet polish etchant HNA (HF, Nitric Acid, and Acetic Acid 2:15:5) [11] with photoresist and wax composite protection layer. After the polish etching was finished, the optic tunnels were patterned in photoresist (AZ4620) on the backside of the MUMPs chip by precise double-sided alignment. After lithography, the sample was placed into a 4-inch carrier wafer for the DRIE. The carrier wafer can hold the MUMPs chip during the processes under pressurized helium. It is used to provide heat transfer between the chip and the coolant with good temperature uniformity. The carrier wafer also prevents the active radical plasma damaging the front-side MUMPs devices. The through-hole etch stops at the bottom of the nitride layer. A following Trion RIE $\mathrm{SF}_{6}$ plasma etch made the optical channel more positive, allowing the use of large NA microlenses, and thus reducing our focal length to make the TMOS module more compact. $\mathrm{SF}_{6}$ plasma will slowly remove the nitride layer and also the Poly0 layer. When the Poly0 on the detection structure has been removed, the etching stops at the bottom of the first oxide layer. After RIE etching, sacrificial oxide layers were removed by concentrated $49 \% \mathrm{HF}$ and released by carbon dioxide critical point drying.
In order to prevent in-use stiction, besides adding dimples under the structure and bumps for the sidewall, surface modifications such as PECVD hydrophobic fluorocarbon polymer coating has been used. The low surface-energy, fluorocarbon films are a good solid lubricant. The fluorocarbon film is also used as electrical insulator between the electrostatic actuator and the electrode. After the MUMPs chip was removed from the PECVD chamber, it was ready for testing.

\section{EXPERIMENTAL RESULTS}

Experimentally, a pair of zigzag-actuators controls an $18 \mu \mathrm{m} \times 22 \mu \mathrm{m}$ opening at $38-130 \mathrm{~V}$ with large controllable static displacement, depending on the zigzag geometry and zigzag electrode thickness combination (Table 1).

Table 1. Dimensions of different zigzag designs (widths were measured by SEM). 4 designs were tested in 2 MUMPs runs each. Voltage displacement results are shown in Figure 6. Results suggest that the pull-in effect depends on the width (i.e., stiffness), with stiffer zigzags having a larger range of controllable motion.

\begin{tabular}{|c|c|c|c|c||}
\hline MUMPS Run \# & $44 / 45$ & $44 / 45$ & $48 / 49$ & $48 / 49$ \\
\hline Design & Type1 & Type2 & Type3 & Type4 \\
\hline Zigzag width $(\mu \mathrm{m})$ & 1.5 & 3.0 & 1.8 & 2.0 \\
\hline Zigzag thickness $(\mu \mathrm{m})$ & 1.5 & 3.5 & 3.5 & 3.5 \\
\hline Electrode height $(\mu \mathrm{m})$ & 3.5 & 3.5 & 3.5 & 3.5 \\
\hline Number of teeth & 10 & 10 & 11 & 11 \\
\hline Natural frequency $(\mathrm{kHz})$ & $<6.6$ & & $12.6-15.4$ & $16.6-18.6$ \\
\hline
\end{tabular}

We observed natural frequencies for single actuators ranging from 5.2 to $18.6 \mathrm{kHz}$. Optical test results showed that the shutter can effectively turn the light beam on and off with very high contrast ratios (Figure 7).

Understanding the failure modes is critical for the reliability of these actuators. This includes optical failure, mechanical failure, and electrical failure. Optical failures originate mainly from over-etching or misalignment during throughhole-etching. Mechanical failures are caused by actuators being stuck because of contamination, levitation, electrodedamage and shutter failure. The main electrical failure is short circuit. Depositing PECVD fluorocarbon $\left(\mathrm{C}_{3} \mathrm{~F}_{6}\right)$ polymer antisticking and isolation conformal coating of the actuators $(0.28 \mu \mathrm{m}$ on top) yields a breakdown voltage up to 140V. Careful design and a well-controlled process can avoid all of these failure modes.

Fatigue and reliability testing is currently ongoing: for a single zigzag actuator (Type 3 ), after $>7.6 \times 10^{9}$ cycles under resonance frequency $(12.6 \mathrm{kHz}$, amplitude $15 \mu \mathrm{m}$, sinusoid excitation, $\left.V_{\min }=-48 \mathrm{~V}, V_{\max }=48 \mathrm{~V}\right)$, no fatigue or frequency shifts have been observed. 


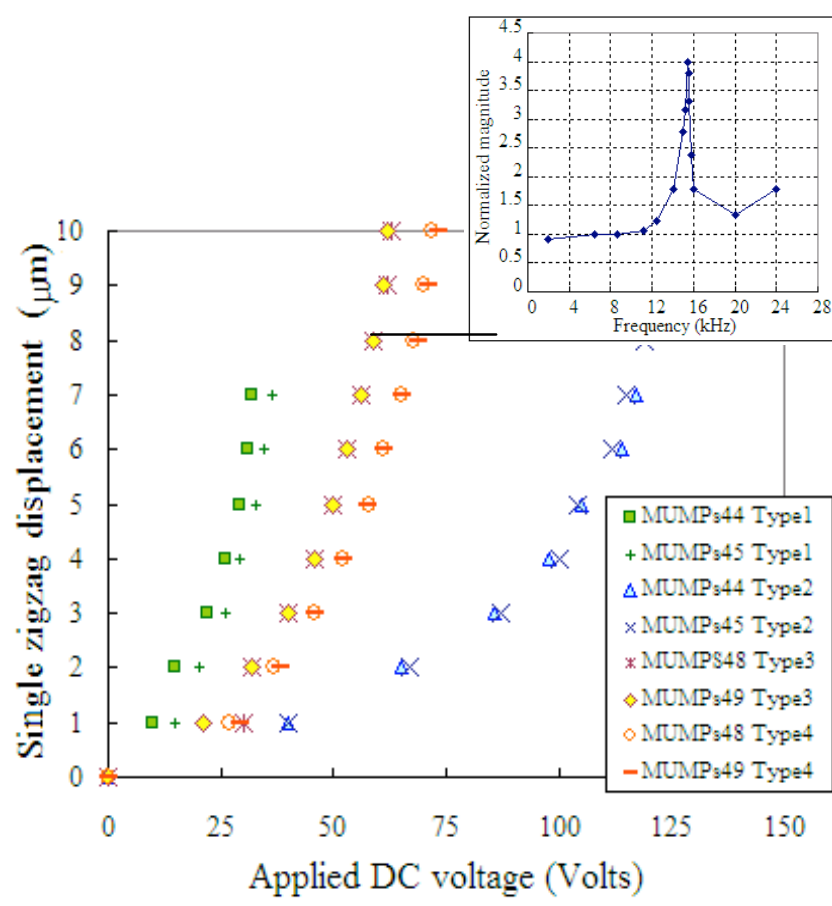

Figure 6. The experimental results show that the displacement of zigzags before pull-in is proportional to voltage squared and inverse to zigzag area moment of inertia under similar geometric conditions (same number of teeth). Each type of design has been tested on at least two MUMPs runs. It shows good reproducibility of performance. The figure in the upper-right corner represents the frequency response of design Type 3.

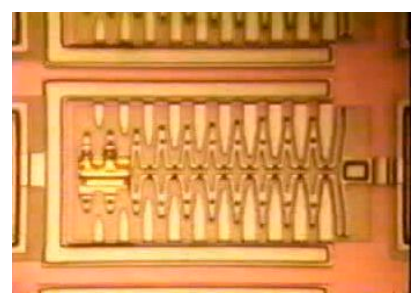

(a) Bright field switch closed

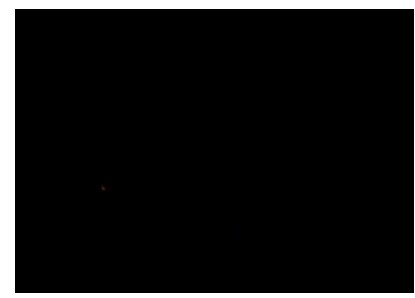

(c) Dark field switch closed

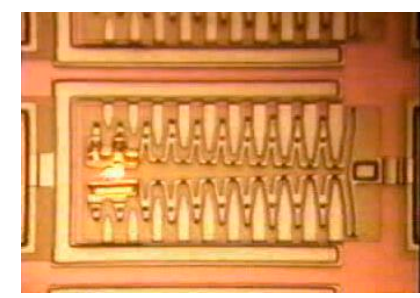

(b) Bright field switch open

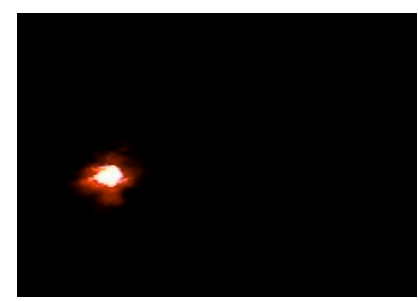

(d) Dark field switch open
Figure 7. Optical test results show that TMOS can achieve near-ideal contrast ratio.

For the dual actuator TMOS (Type4), after $>1.78 \times 10^{9}$ cycles ( $2 \mathrm{kHz}$, full-range-switching-mode), no defects or shutter damage caused by collision were apparent. The precise shutter movement was monitored by strobe-integrated microscopy. The bounce caused by shutter collision was less than $1 \mu \mathrm{m}$. The testing proceeded under ambient room atmosphere and temperature and demonstrated reliable and repeatable performance of the highly space-efficient electrostatic actuators.

\section{CONCLUSIONS}

We have demonstrated highly space-efficient electrostatic actuators with large static lateral displacement for TMOS, and a DRIE post-process for MUMPS ${ }^{\circledR}$ chips. With their size, speed, reliable and repeatable performance, the TMOS is not only the heart of our continuing effort for transmissive displays but also very attractive to other possible MOEMS applications such as pocketable projectors, optic data storage devices and highly dense optic switch arrays for networks.

\section{REFERENCES}

[1] L.J. Hornbeck, W.E. Nelson, "Bistable deformable mirror device," OSA Technical Digest Series, Vol. 8, Spatial Light Modulators and Applications, p.107, 1988.

[2] L.J. Hornbeck, "Digital light processing for highbrightness, high-resolution applications," Electronic imaging, projection displays III, 10-12 February 1997, San Jose, California, USA.

[3] R.W. Corrigan, D.T. Amm, C.S. Gudeman, "Grating light valve technology for projection displays," International Display Workshop, Kobe, Japan, Paper Number LAD5-1, 9 Dec 1998.

[4] S. Bains, "Micromechanical display use interferometric modulation," Optical engineering report SPIE, no. 199, July 2000.

[5] http://www.parc.xerox.com/dhl/projects/gyricon/

[6] W.H. Juan, S.W. Pang, "High-Aspect Ratio Si Vertical Micromirror Array for Optical Switching," J. of MEMS, vol.7, no.2, pp.207-212, 1998.

[7] G. Perregaux, S. Gonseth, P. Debergh, J. P. Thiebaud, H. Vuilliomenet, "Arrays of Addressable High-Speed Optical Microshutters”, Proc. IEEE MEMS, pp.232-235, 2001.

[8] R. Legtenberg, J. Gilbert, S.D. Senturia, "Electrostatic Curved Electrode Actuators", J. of $M E M S$, vol.6, no.3, pp.257-265, 1997.

[9] J.D. Grade, H. Jerman, T.W. Kenny, “A large-deflection electrostatic actuator for optical switching application", pp.97-100, Sensor and Actuator Workshop, Hilton Head Island, June 4-8, 2000.

[10] W. Ye, S. Mukherjee, N.C. MacDonald, "Optimal Shape Design of an Electrostatic Comb Drive in Microelectromechanical System", J. of MEMS, pp.16-25, v.67, no.1, 1998.

[11] B. Schwartz, H. Robbins, "Chemical etching of Silicon, IV," Etching technology, J. Electrochem. Soc. 123, 1903, 1967. 